\title{
Asymptotic Solution for a Class of Epidemic Contagion Ecological Nonlinear Systems
}

\author{
Yi-Hu Feng $\mathbb{D}^{1,2}$ and Lei Hou ${ }^{1}$ \\ ${ }^{1}$ Department of Mathematics, Shanghai University, Shanghai 200444, China \\ ${ }^{2}$ Department of Electronics and Information Engineering, Bozhou University, Bozhou, Anhui 236800, China
}

Correspondence should be addressed to Yi-Hu Feng; fengyihubzxy@163.com

Received 4 October 2019; Accepted 6 May 2020; Published 23 May 2020

Academic Editor: Gisele Mophou

Copyright (c) 2020 Yi-Hu Feng and Lei Hou. This is an open access article distributed under the Creative Commons Attribution License, which permits unrestricted use, distribution, and reproduction in any medium, provided the original work is properly cited.

In this paper, a class of systems for epidemic contagion is considered. An epidemic virus ecological model is described. Using the generalized variation iteration method, the corresponding approximate solution to the nonlinear system is obtained and the method for this approximate solution is pointed out. The accuracy of approximate solution is discussed, and it can control the epidemic virus transmission by using the parameters of the system. Thus, it has the value for practical application.

\section{Introduction}

Many results on epidemic virus transmission have been studied in the medical science and ecological environment [1-4]. There is a model of the nonlinear differential system for its basic physical phenomena. Currently, the solving method of the nonlinear epidemic virus population system is an important field [5-8].

The nonlinear differential system is an attractive investigated subject. Adams et al. considered the possible "shock" patterns that can exist in the solution to a singular perturbed, third-order nonlinear ordinary differential equation arising as the travelling-wave reduction of the Kuramoto-Sivashinsky equation. In particular, the existence (or otherwise) of oscillatory shocks and multiple shocks made up of combinations of oscillatory and monotonic shocks is examined, using an optimal truncation strategy to track crucial exponential small terms lying beyond all orders of the (divergent) algebraic expansion. The results provide further understanding of numerical solutions previously obtained by others, as well as giving a methodology which is applicable to much broader classes of differential equations exhibiting multiscale phenomena; they also afford the same new insight into the multiscales technique [9]. Bell and Deng studied singular perturbation of $\mathrm{N}$-front traveling waves in the Fitzhugh-Nagumo equations [10]. Many approximate methods have been developed, such as the boundary layer method, the matched asymptotic method, and the multiple scales method. Many scholars have performed a great deal of work [11-21]. The authors also studied a class of ecological environment systems, such as HIV transmission, the solitary wave, the reaction diffusion system, and the model of prey and predator [22-28].

He proposed a novel variational approach for limit cycles of a kind of nonlinear oscillators. Some examples are given to illustrate the effectiveness and convenience of the method. The obtained results are valid for the whole solution domain with high accuracy [29]. Tao et al. discussed the variational approach and the Hamiltonian approach to nonlinear oscillators systematically. They gave an extension of the methods to a singular nonlinear oscillator by the iteration perturbation method [30]. In this paper, using the generalized variational iteration method, we study a class of epidemic virus population systems.

The rest of this paper is organized as follows. In Section 2 , we studied a class of epidemic virus transmission population systems and then introduced the names of the variables in the equation and the boundary conditions. In Section 3, we constructed the generalized variation iterations, obtained the first approximations, used the same method, from the generalized variation iterations and the variation principle, and obtained higher-order approximate solution sequences to the system, and these sequences converge uniformly. In Section 4, we gave an example, using 
the generalized variation iteration method and we obtained the approximate solution to the system. In Section 5, we solved asymptotic expansions to the epidemic virus transmission population dynamic system by using the perturbation method. Comparing the obtained approximate solutions by using two methods, we see that the approximate solution to the epidemic virus transmission population dynamic system by using the method of the generalized functional variational iteration possesses a good accuracy. In Section 6, we considered the virus transmission parameters, obtained the simulated curves for the approximate solution, and took measures to control the virus transmission. In Section 7, we obtained a conclusion, simplified the basic model, and solved it by using the approximate method. The method of the generalized variational iteration is simple and valid.

\section{Epidemic Virus Population System}

The following is a class of epidemic virus transmission population systems $[5,6]$ :

$$
\begin{aligned}
\frac{d S}{d t}-\alpha+\beta K(S) I+a S & =0, \\
\frac{d I}{d t}-\beta K(S) I+(a+b+c) I-d R & =0, \\
\frac{d R}{d t}-\gamma K(S) I+(a+e+d) R & =0,
\end{aligned}
$$

where $S, I$, and $R$ are the numbers of the sufferer population, susceptible population, and potential population in the broken area, respectively, $t$ is the time, $\alpha$ is an immigrate rate of the sufferer population, $a$ is althe natural death rate, $c$ and $e$ are the death rate of susceptible and potential populations, respectively, $b$ is the change rate of the sufferer population changed into the potential population, and $d$ is the lost rate of immunity in potential population. The abovementioned $e$ is a nonnegative constant, other parameters are positive constants, $a+b+c>d$, and $\beta K(S)$ and $\gamma K(S)$ are nonlinear infectiousness for the sufferer population and potential population, respectively. Also, $K(S)$ is a sufficiently smooth function in $(-\infty, \infty)$. Specially, nonlinear function $K(S)$ possesses the following bounds:

$$
\begin{gathered}
a S^{b}, b>0 ; \\
\frac{b S^{n}}{a+S^{n}}, a, b>0, n \geq 1 ; \\
a(1-\exp (-b S)), a, b>0 ; \\
\frac{a S}{1+b S+\sqrt{1+2 b S}}, a, b>0 ; \\
\frac{1-\exp (-b S)}{1+\operatorname{eexp}(-b S)}, a, b>0,
\end{gathered}
$$

and so on.

\section{Generalized Variation Iteration System}

In order to obtain the approximate solution to systems (1)-(3), we introduce a set of functional $F_{i}(i=1,2,3)$ $[27,28]$ :

$$
\begin{aligned}
& F_{1}=S-\int_{0}^{t} \lambda_{1}(\tau)\left[\frac{d S}{d \tau}-\alpha+\beta K(\widetilde{S}) \widetilde{I}+a S\right] d \tau \\
& F_{2}=I-\int_{0}^{t} \lambda_{2}(\tau)\left[\frac{d I}{d \tau}-\beta K(\widetilde{S}) \widetilde{I}+(a+b+c) I-d \widetilde{R}\right] d \tau \\
& F_{3}=R-\int_{0}^{t} \lambda_{3}(\tau)\left[\frac{d R}{d \tau}-\gamma K(\widetilde{S}) \widetilde{I}+(a+d+e) R\right] d \tau
\end{aligned}
$$

where $\widetilde{S}, \widetilde{I}$, and $\widetilde{R}$ are the restricted variables of $S, I$, and $R$, respectively, while $\lambda_{i} \quad(i=1,2,3)$ are the Lagrange multiplicators.

Compute the variations $\delta F_{i}(i=1,2,3)$ of functional and equate to zero as follows:

$$
\begin{aligned}
& \delta F_{1}=\delta S-\left.\left(\lambda_{1} \delta S\right)\right|_{\tau=t}+\int_{0}^{t}\left(\frac{\partial \lambda_{1}}{\partial \tau}+a \lambda_{1}\right) \delta S d \tau=0 \\
& \delta F_{2}=\delta I-\left.\left(\lambda_{2} \delta I\right)\right|_{\tau=t}+\int_{0}^{t}\left(\frac{\partial \lambda_{2}}{\partial \tau}+(a+b+c) \lambda_{2}\right) \delta I d \tau=0 \\
& \delta F_{3}=\delta R-\left.\left(\lambda_{3} \delta R\right)\right|_{\tau=t}+\int_{0}^{t}\left(\frac{\partial \lambda_{3}}{\partial \tau}+(a+d+e) \lambda_{3}\right) \delta R d \tau=0
\end{aligned}
$$

From equations (8)-(10), we have

$$
\begin{array}{r}
\frac{\partial \lambda_{1}}{\partial \tau}+a \lambda_{1}=0, \\
\frac{\partial \lambda_{2}}{\partial \tau}+(a+b+c) \lambda_{2}=0, \\
\frac{\partial \lambda_{3}}{\partial \tau}+(a+d+e) \lambda_{3}=0, \\
\left.\lambda_{i}(\tau)\right|_{\tau=t}=1, \quad i=1,2,3 .
\end{array}
$$

From problems (11) and (12), we obtain

$$
\begin{aligned}
& \lambda_{1}(t)=\exp \{a(t-\tau)\}, \\
& \lambda_{2}(t)=\exp \{(a+b+c)(t-\tau)\}, \\
& \lambda_{3}(t)=\exp \{(a+d+e)(t-\tau)\} .
\end{aligned}
$$

From equations (5)-(7) and (13)-(15), we can construct the following generalized variation iterations: 


$$
\begin{aligned}
S_{n+1} & =S_{n}-\int_{0}^{t} \exp \{a(t-\tau)\}\left[\frac{d S_{n}}{d \tau}-\alpha+\beta K\left(S_{n}\right) I_{n}+a S_{n}\right] d \tau, \quad n=0,1, \ldots, \\
I_{n+1} & =I_{n}-\int_{0}^{t} \exp \{(a+b+c)(t-\tau)\}\left[\frac{d I_{n}}{d \tau}-\beta K\left(S_{n}\right) I_{n}+(a+b+c) I_{n}-d R_{n}\right] d \tau, \quad n=0,1, \ldots \\
R_{n+1} & =R_{n}-\int_{0}^{t} \exp \{(a+d+e)(t-\tau)\}\left[\frac{d R_{n}}{d \tau}-\gamma K\left(S_{n}\right) I_{n}+(a+d+e) R_{n}\right] d \tau, \quad n=0,1, \cdots
\end{aligned}
$$

First, we select a set of initial approximations $\left(S_{0}(t), I_{0}(t), R_{0}(t)\right)$. Also, it is a set of solutions to the linear system as follows:

$$
\begin{aligned}
& \frac{d S_{0}}{d t}=\alpha-a S_{0}, \\
& \frac{d I_{0}}{d t}=-(a+b+c-d) I_{0}+d R_{0}, \\
& \frac{d R_{0}}{d t}=-(a+d+e) R_{0} .
\end{aligned}
$$

It is easy to obtain a set of solutions to systems (19)-(21), so we have

$$
\begin{aligned}
& S_{0}(t)=C_{1}^{0} \exp (-a t)+\alpha t, \\
& I_{0}(t)=C_{2}^{0} \exp \{-(a+b+c-d) t\}-\frac{d C_{3}^{0}}{a+d+e} \exp \{-(a+d+e) t\}
\end{aligned}
$$

$R_{0}(t)=C_{3}^{0} \exp \{-(a+d+e) t\}$

where $C_{i}^{0}(i=1,2,3)$ are the arbitrary constants, which can be decided by the conditions of systems (1)-(3).

From equations (16)-(18), we can obtain the first approximations:

$$
\begin{aligned}
S_{1}(t)= & C_{1}^{0} \exp (-a t)+\alpha t-\beta \int_{0}^{t} \exp \{a(t-\tau)\}\left[K\left(C_{1}^{0} \exp (-a \tau)+\alpha \tau\right)\right. \\
& \left.\times\left(C_{2}^{0} \exp \{-(a+b+c-d) \tau\}-\frac{d C_{3}^{0}}{(a+d+e)} \exp \{-(a+d+e) \tau\}\right)\right] d \tau \\
I_{1}(t)= & C_{2}^{0} \exp \{-(a+b+c-d) t\}-\frac{d C_{3}^{0}}{(a+d+e)} \exp \{-(a+d+e) t\}+\beta \int_{0}^{t} \exp \{(a+b+c)(t-\tau)\}\left[K\left(C_{1}^{0} \exp (-a \tau)+\alpha \tau\right)\right. \\
& \left.\times\left(C_{2}^{0} \exp \{-(a+b+c-d) \tau\}-\frac{d C_{3}^{0}}{(a+d+e)} \exp \{-(a+d+e) \tau\}\right)\right] d \tau \\
R_{1}(t)= & C_{3}^{0} \exp \{-(a+d+e) t\}+\gamma \int_{0}^{t} \exp \{(a+d+e)(t-\tau)\}\left[K\left(C_{1}^{0} \exp (-a \tau)+\alpha \tau\right)\right. \\
& \left.\times\left(C_{2}^{0} \exp \{-(a+b+c-d) \tau\}-\frac{d C_{3}^{0}}{(a+d+e)} \exp \{-(a+d+e) \tau\}\right)\right] d \tau .
\end{aligned}
$$

Using the same method, from generalized variation iterations (16)-(18), we can obtain higher-order approximate solution to systems (1)-(3).

From the generalized variation iterations (16)-(18) and the variation principle, we can obtain sequences $\left\{S_{n}(t)\right\},\left\{I_{n}(t)\right\}$, and $\left\{R_{n}(t)\right\}$. Also, these sequences converge uniformly [31, 32].
Let

$$
\begin{aligned}
S(t) & =\lim _{n \longrightarrow \infty} S_{n}(t), \\
I(t) & =\lim _{n \longrightarrow \infty} I_{n}(t), \\
R(t) & =\lim _{n \longrightarrow \infty} R_{n}(t) .
\end{aligned}
$$

Thus, we have the following theorem. 
Theorem 1. If $K(S)$ is a sufficiently smooth bounded function in $(-M, M)$, where $M$ is a sufficiently large constant, and

$$
\begin{gathered}
a S^{b}, b>0, \\
\frac{b S^{n}}{a+S^{n}}, a, b>0, n \geq 1, \\
a(1-\exp (-b S)), a, b>0, \\
\frac{a S}{1+b S+\sqrt{1+2 b S}}, a, b>0, \\
\frac{1-\exp (-b S)}{1+\exp (-b S)}, a, b>0,
\end{gathered}
$$

then $(S(t), I(t), R(t))$ is the exact solution to systems (1)-(3) in $(-M, M)$.

\section{Example}

For convenience, from (1)-(3), we set that $a=b=c=d=$ $\alpha=\delta=1, \quad e=0,0<\beta=\gamma=\varepsilon \ll 1, \quad$ and $K(S)=S$. Thus, we consider the following example:

$$
\begin{aligned}
\frac{d S}{d t}-1+S+\varepsilon I S & =0, \\
\frac{d I}{d t}+3 I-R-\varepsilon I S & =0, \\
\frac{d R}{d t}+2 R-\varepsilon I S & =0 .
\end{aligned}
$$

Using the generalized variation iteration method, we can obtain the approximate solution to systems (30)-(32).

From equations (22)-(24), we have the initial approximate solution $\left(S_{0}(t), I_{0}(t), R_{0}(t)\right)$ to systems (30)-(32). Also, we have

$$
\begin{aligned}
& S_{0}(t)=\exp (-t)+t, \\
& I_{0}(t)=\frac{1}{2} \exp (-2 t), \\
& R_{0}(t)=\exp (-2 t) .
\end{aligned}
$$

where we can take $C_{i}^{0}=1, i=1,2,3$.

From equations (25)-(27) and (33) and (34), we can obtain the first approximations:

$$
\begin{aligned}
& S_{1}(t)=\exp (-t)+t-\varepsilon \int_{0}^{t} \exp (t-\tau)\left[(\exp (-\tau)+\tau)\left(\frac{1}{2} \exp (-2 \tau)+1\right)\right] d \tau \\
& I_{1}(t)=\frac{1}{2} \exp (-2 t)+\varepsilon \int_{0}^{t} \exp \{3(t-\tau)\}\left[(\exp (-\tau)+\tau)\left(\frac{1}{2} \exp (-2 \tau)+1\right)\right] d \tau \\
& R_{1}(t)=\exp (-2 t)+\varepsilon \int_{0}^{t} \exp \{2(t-\tau)\}\left[(\exp (-\tau)+\tau)\left(\frac{1}{2} \exp (-2 \tau)+1\right)\right] d \tau
\end{aligned}
$$

Using the same method, from generalized variation iterations (33)-(35), we can obtain the higher-order approximate solution $\left(S_{n}(t), I_{n}(t), R_{n}(t)\right)(n=2,3, \cdots)$ to systems (5)-(7), and their constructions are omitted.

\section{Accuracy of Approximate Solution}

Now, we assume the absolute nondimensional parameters to systems (30)-(32). From Figures 1-3, we show the simulated curves: the real curve is the exact solution $(S(t), I(t), R(t))$ and the approximation curve is the first approximation solution $\left(S_{1}(t), I_{1}(t), R_{1}(t)\right)$.

On the other hand, we can also solve asymptotic expansions to epidemic virus transmission population dynamic systems (30)-(32) by using the perturbation method.
Let the solution $(\bar{S}, \bar{I}, \bar{R})$ to systems (30)-(32) have the following form:

$$
\begin{aligned}
& \bar{S}=\sum_{n=0}^{\infty} \bar{S}_{n}(t) \varepsilon^{n}, \\
& \bar{I}=\sum_{n=0}^{\infty} \bar{I}_{n}(t) \varepsilon^{n}, \\
& \bar{R}=\sum_{n=0}^{\infty} \bar{R}_{n}(t) \varepsilon^{n}, \\
& 0<\varepsilon \ll 1 .
\end{aligned}
$$

Substituting equation (39) into systems (30)-(32), developing nonlinear terms in $\varepsilon$, equating coefficients of the same powers of $\varepsilon$, and letting initial values be $(S(0), I(0), R(0))=$ 


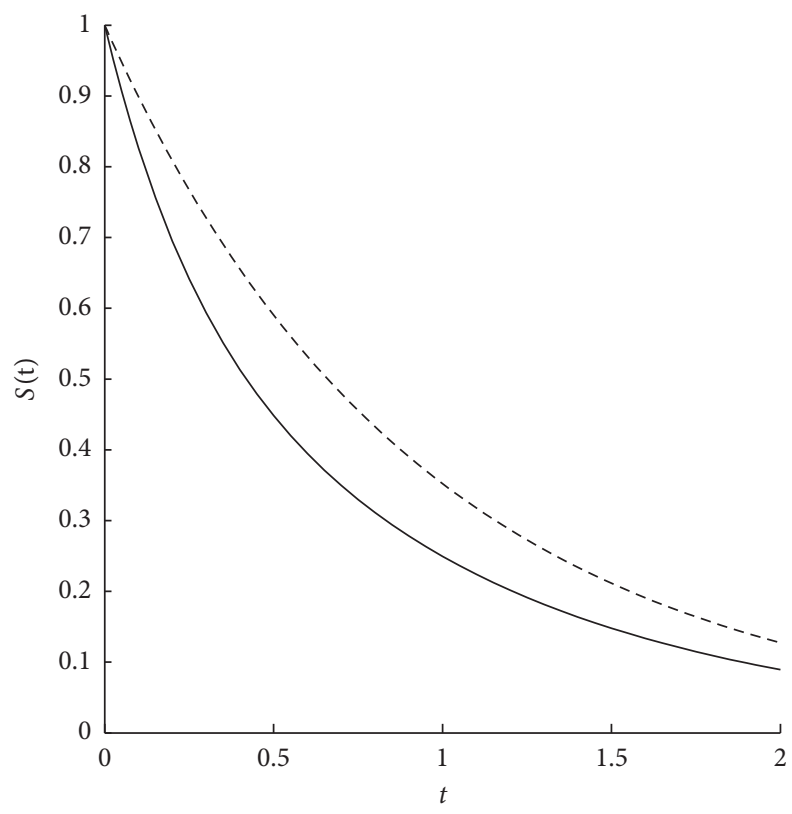

FIGURE 1: Illustration for the curves of $S(t)$ (real curve) and $S_{1}(t)$ (approximation curve).

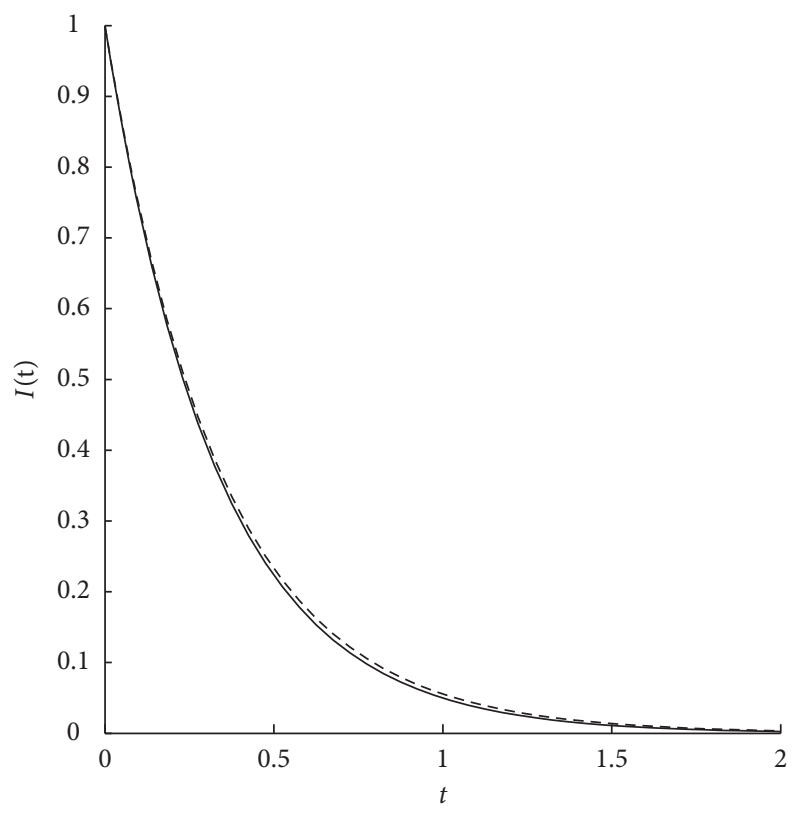

FIgURE 2: Illustration for the curves of $I(t)$ (real curve) and $I_{1}(t)$ (approximation curve).

$(1,1,1)$ for systems $(30)-(32)$, we can obtain $\bar{S}_{n}, \bar{I}_{n}, \bar{R}_{n}(n=$ $0,1, \cdots)$ successively. For $n=0$, we have

$$
\begin{aligned}
& \bar{S}_{0}(t)=\exp (-t)+t, \\
& \bar{I}_{0}(t)=\frac{1}{2} \exp (-2 t),
\end{aligned}
$$

$$
\bar{R}_{0}(t)=\exp (-2 t)
$$

Substituting equations (39)-(42) into expansions (30)-(32), we obtain the first-order perturbed solution $\left(\bar{S}_{1 a p p}, \bar{I}_{1 a p p}, \bar{R}_{1 a p p}\right)$ to systems (30)-(32): 


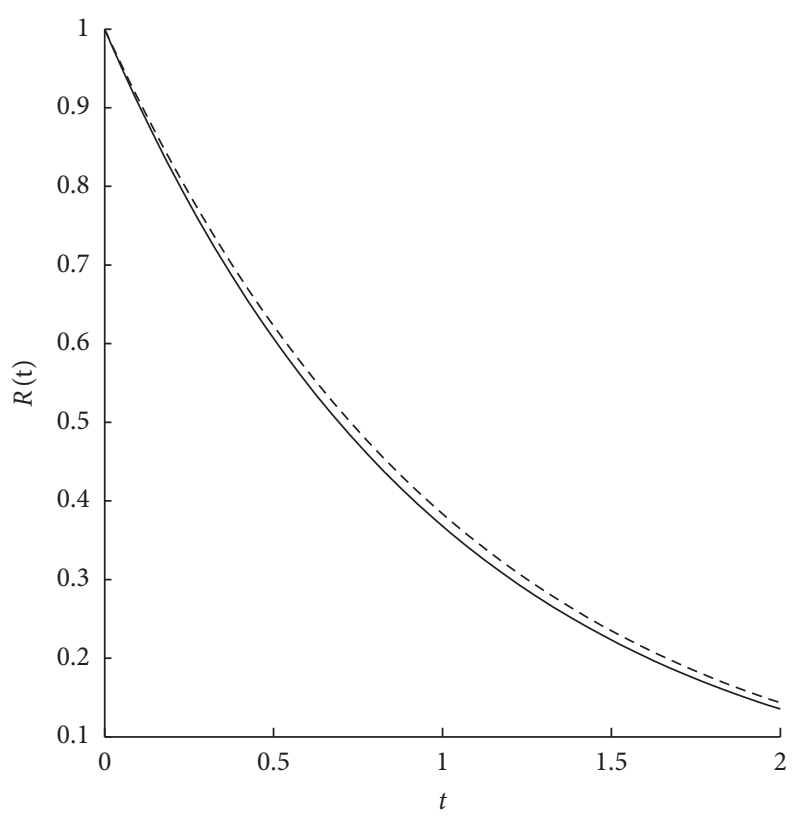

FIgURE 3: Illustration for the curves of $R(t)$ (real curve) and $R_{1}(t)$ (approximation curve).

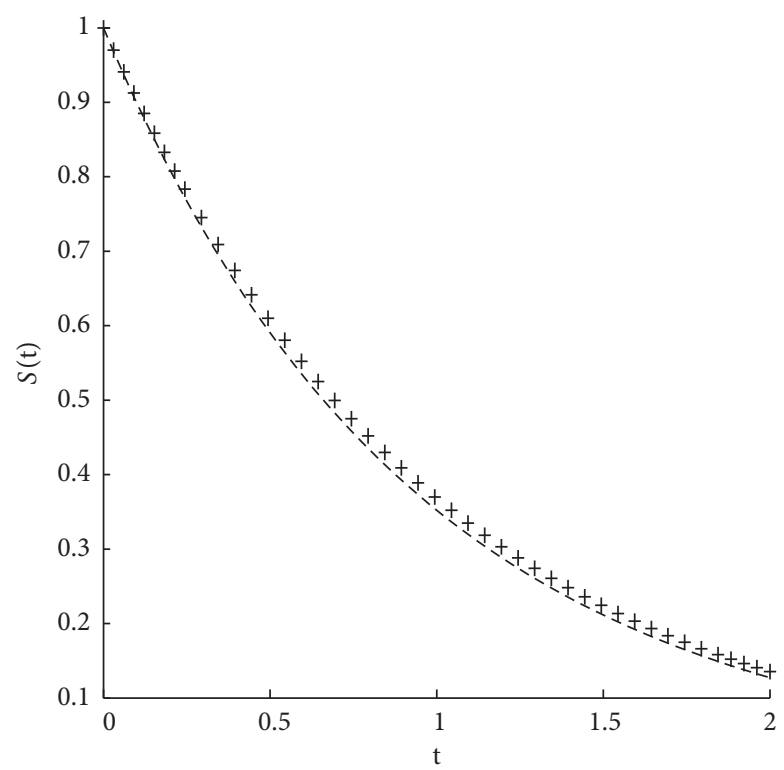

Figure 4: Illustration for the curve of $S(t)$ (real curve as $\beta=\gamma=0.5$ and ' $+{ }^{\prime}$ curve as $\beta=\gamma=5$ ).

$$
\begin{aligned}
& \bar{S}_{1 \text { app }}(t)=\exp (-t)+t-\varepsilon \int_{0}^{t} \exp (t-\tau)\left[(\exp (-\tau)+\tau)\left(\frac{1}{2} \exp (-2 \tau)+1\right)\right] d \tau+O\left(\varepsilon^{2}\right), \quad 0<\varepsilon \ll 1, \\
& \bar{I}_{1 \text { app }}(t)=\exp (-2 t)-\frac{1}{2} \exp (-2 t)+\varepsilon \int_{0}^{t} \exp \{3(t-\tau)\}\left[(\exp (-\tau)+\tau) \times\left(\frac{1}{2} \exp (-2 \tau)+1\right)\right] d \tau+O\left(\varepsilon^{2}\right), \quad 0<\varepsilon \ll 1, \\
& \bar{R}_{1 a p p}(t)=\exp (-2 t)+\varepsilon \int_{0}^{t} \exp \{2(t-\tau)\}\left[(\exp (-\tau)+\tau) \times\left(\frac{1}{2} \exp (-2 \tau)+1\right)\right] d \tau+O\left(\varepsilon^{2}\right), \quad 0<\varepsilon \ll 1
\end{aligned}
$$

Comparing obtained approximate solutions (36)-(38) with (43)-(45) by using the above two methods, respectively, we observe that they are the same for the first-order precision $O(\varepsilon), 0<\varepsilon \ll 1$. Therefore, we can see that the 


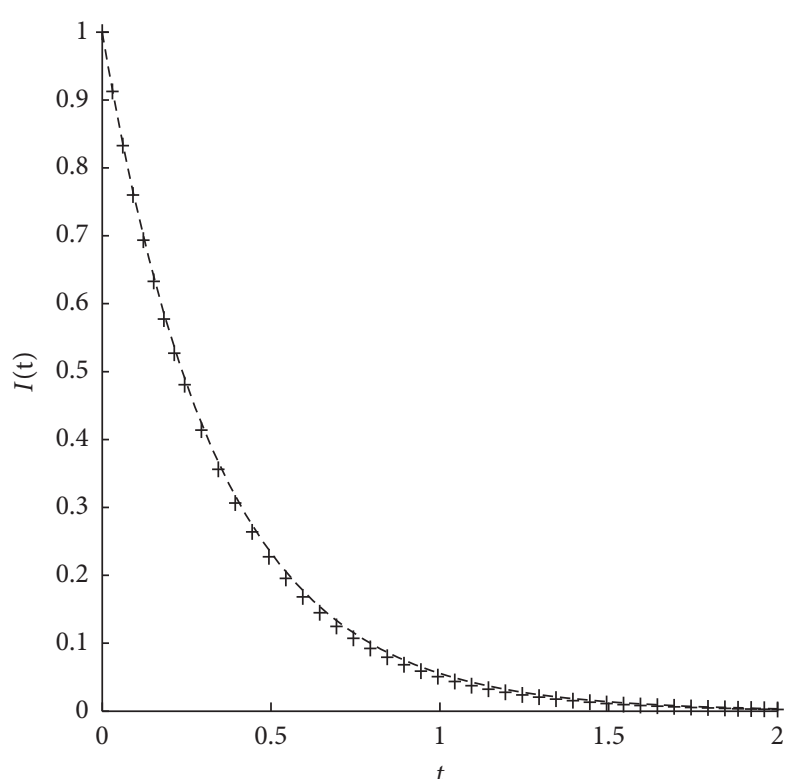

FIGURE 5: Illustration for the curve of $I(t)$ (real curve as $\beta=\gamma=0.5$ and ' + ' curve as $\beta=\gamma=5$ ).

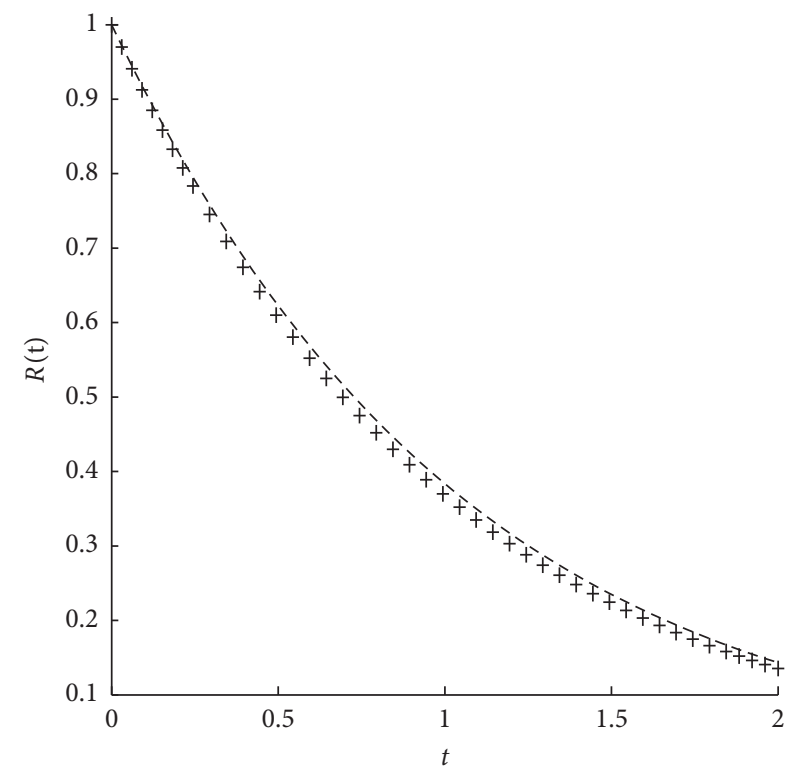

Figure 6: Illustration for the curve of $R(t)$ (real curve as $\beta=\gamma=$ 0.5 and ' + ' curve as $\beta=\gamma=5$ ).

approximate solution $(\bar{S}(t), \bar{I}(t), \bar{R}(t))$ to epidemic virus transmission population dynamic systems (30)-(32) by using the method of the generalized functional variational iteration possesses a good accuracy.

\section{Change Virus Transmission Parameters}

We can obtain the simulated curves for the approximate solution and take measures to control the virus transmission.

Now, we assume the absolute nondimensional parameters of systems (25)-(27) as follows:

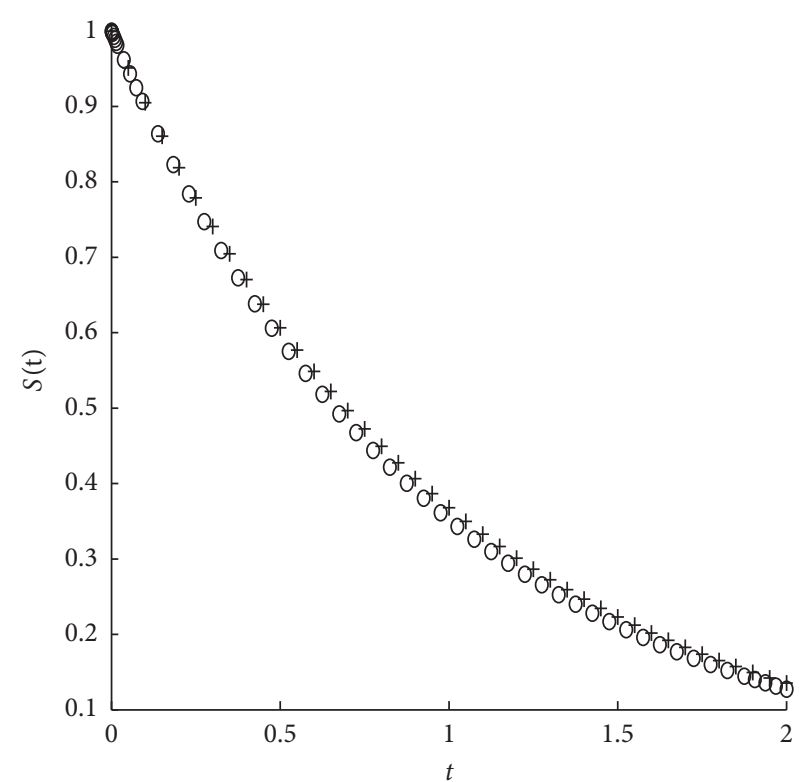

FIgURE 7: Illustration for the curve of $S(t)$ (real curve as $K(S)=S$, and ' $o$ ' curve as $K(S)=S^{5}$ ).

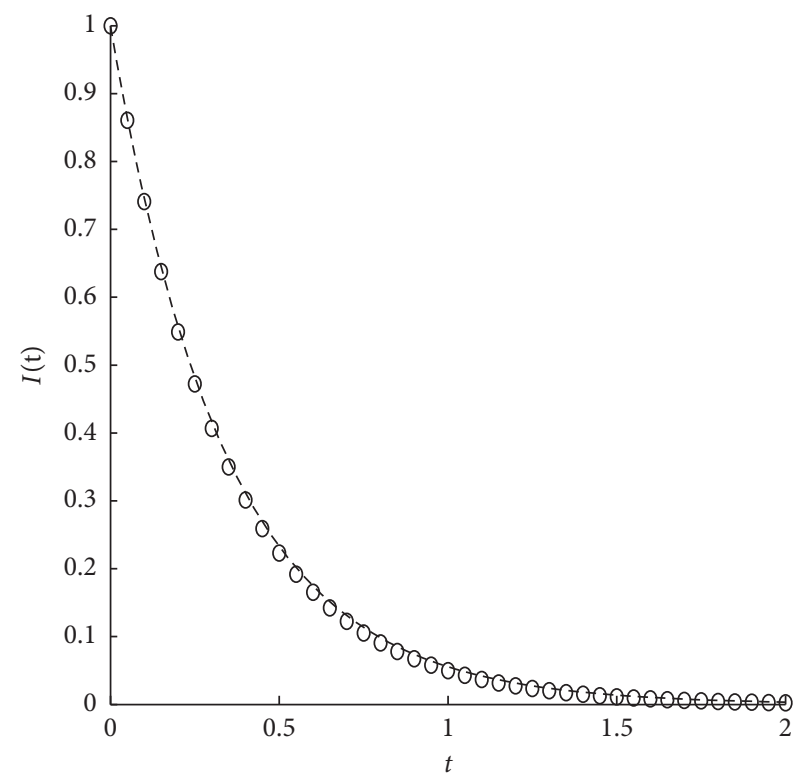

FIgURE 8: Illustration for the curve of $I(t)$ (real curve as $K(S)=S$, and ' $O$ ' curve as $K(S)=S^{5}$ ).

(1) Take the virus transmission parameters as $a=b=$ $c=1, \alpha=d=e=0, \beta=\gamma=0.5$ or $\beta=\gamma=5$, and $K(S)=S$, respectively. We can obtain the simulated curves $S(t), I(t)$, and $R(t)$ (see Figures 4-6). Also, we show the simulated curves of $S(t), I(t)$, and $R(t)$, respectively, where the real curve is the case as $\beta=$ $\gamma=0.5$ and the ${ }^{\prime}+{ }^{\prime}$ curve is the case as $\beta=\gamma=5$.

(2) Take the virus transmission parameters as $a=b=c=1, \alpha=d=e=0, \beta=\gamma=5$, and $K(S)=$ $S$ or $K(S)=S^{5}$, respectively. We can obtain the simulated curves $S(t), I(t)$, and $R(t)$ (see 


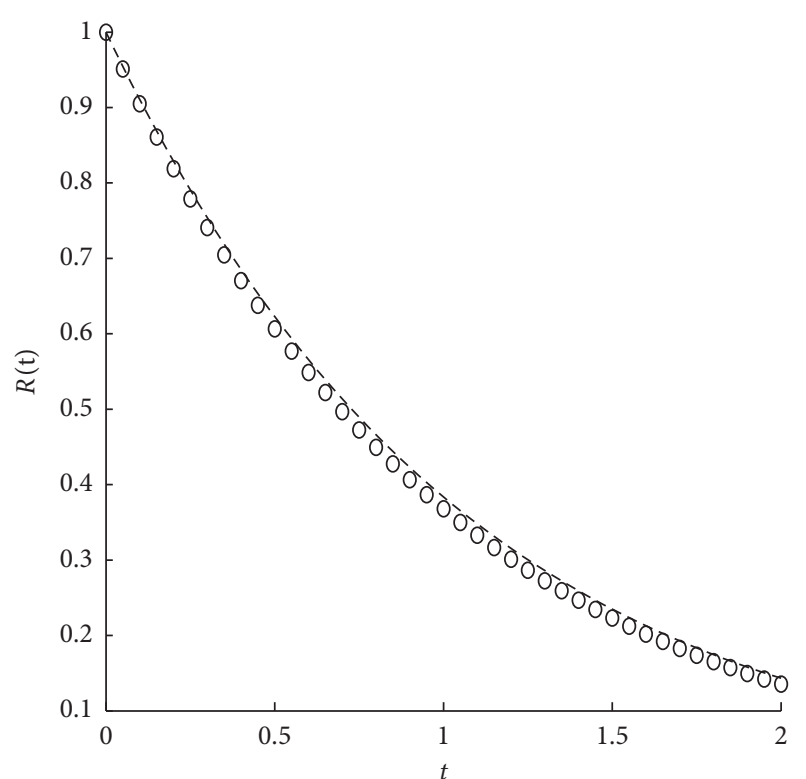

FIGURE 9: Illustration for the curve of $R(t)$ (real curve as $K(S)=S$, and 'o' curve as $K(S)=S^{5}$ ).

Figures 7-9). Also, we show the simulated curves of $S(t), I(t)$, and $R(t)$, respectively, where the real curve is the case as $K(S)=S$ and the ${ }^{\prime}+{ }^{\prime}$ curve is the case as $K(S)=S^{5}$.

Thus, from Figures 4-9, we can use the parameters to control the virus transmission.

\section{Conclusions}

The epidemic virus transmission is a complicated phenomenon. Hence, we need to simplify the basic model and solve it by using the approximate method. The method of the generalized variational iteration is simple and valid.

The generalized variational iteration is an approximate method, which differs from the general numerical method. The expansion of the solution can also be used analytically. Thus, we can further study the qualitative and quantitative behaviors of the epidemic virus transmission population for the infected and the susceptible populations in the broken area.

\section{Data Availability}

The authors declare that the data in the article are available, shareable, and referenced. Readers can check each article at http://apps.webofknowledge.com/.

\section{Conflicts of Interest}

The authors declare that they have no conflicts of interest.

\section{Authors' Contributions}

Feng Yi-Hu and Hou Lei wrote the original draft.

\section{Acknowledgments}

This research was funded by the National Natural Science Foundation of China under grant no. 11271247; the Key Project of Support Program for Excellent Youth Talent in Colleges and Universities of Anhui Province under grant no. gxyqZD2016520; the Key Project for Natural Science Research of Universities in Anhui Province under grant nos. KJ2015A347, KJ2017A702, and KJ2019A1300; the Key Project for Teaching Research in Anhui Province under grant nos. 2018jyxm0594, 2016jyxm0677, and 2017jyxm0591; the Key Project for Teaching Research of Bozhou University under grant no. 2017zdjy02; and the Key Project for the Natural Science Research of Bozhou University under grant nos. BYZ2017B02 and BYZ2017B03.

\section{References}

[1] R. M. May, "Will a large complex system be stable?" Nature, vol. 238, no. 5364, pp. 413-414, 1972.

[2] P. C. de Ruiter, A.-M. Neutel, and J. C. Moore, "Energetics, patterns of interaction strengths, and stability in real ecosystems," Science, vol. 269, no. 5228, pp. 1257-1260, 1995.

[3] R. M. May, "Biological populations with nonoverlapping generations: stable points, stable cycles, and chaos," Science, vol. 186, no. 4164, pp. 645-647, 1974.

[4] J. M. Hyman, J. Li, and E. A. Stanley, "The differential infectivity and staged progression models for the transmission of HIV," Mathematical Biosciences, vol. 155, no. 1, pp. 77-109, 1999.

[5] H. W. Hethcote and J. W. Vanark, "Modelling HIV transmission and AIDS in the United States," Lecture Notes in Biomath, Springer-Verlag, Berlin, Germany, 1992.

[6] M. X. Liu, Y. H. Ruan, L. T. Han, and Y. C. Zhou, "The summary of dynamic models for HIV transmission," International Journal of Biomathematics, vol. 19, no. 5, pp. 551-560, 2004.

[7] H. N. Moreira and W. Yuquan, "Classroom note:global stability in an \$S \to I \to R Ito I\$ model," SIAM Review, vol. 39, no. 3, pp. 496-502, 1997.

[8] J. J. Chen, "Stability analysis for some epidemic models with nonlinear infections force," International Journal of Biomathematics, vol. 20, no. 3, pp. 286-296, 2005.

[9] K. L. Adams, J. R. King, and R. H. Tew, "Beyond-all-orders effects in multiple-scales symptotic:Travelling-wave solutions to the Kuramoto-Sivashinsky equation," Journal of Engineering Mathematics, vol. 45, no. 1, pp. 197-226, 2003.

[10] D. C. Bell and B. Deng, "Singular perturbation of N-front traveling waves in the Fitzhugh-Nagumo equations," Nonlinear Analysis Real World Applications, vol. 3, no. 4, pp. 515-541, 2003.

[11] R. Suzuki, "Asymptotic behavior of solutions a semilinear heat equation with localized reaction," Advances in Difference Equations, vol. 159, no. 3-4, pp. 283-314, 2010.

[12] M. Ramos, "On singular perturbations of superlinear elliptic systems," Journal of Mathematical Analysis and Applications, vol. 352, no. 1, pp. 246-258, 2009.

[13] T. D'Aprile and A. Pistoia, "On the existence of some new positive interior spike solutions to a semilinear Nuumann problem," Journal of Differential Equations, vol. 248, no. 3, pp. 556-573, 2010.

[14] R. B. Kellogg and N. Kopteva, "A singularly perturbed semilinear reaction-diffusion problem in a polygonal 
domain," Journal of Differential Equations, vol. 248, no. 1, pp. 184-208, 2010.

[15] J. Q. Mo, "Approximate solution of homotopic mapping to solitary wave for generalized nomlinear KdV system," Chinese Physics Letters, vol. 26, no. 1, 2009.

[16] J. Q. Mo, "Variational iteration solving method for a class of generalized Boussinesq equation," Chinese Physics Letters, vol. 26, no. 6, Article ID 060202, 2009.

[17] J. Q. Mo, "Homotopic mapping solving method for gain fluency of a laser pulse amplifier," Science in China Series G: Physics, Mechanics and Astronomy, vol. 52, no. 7, pp. 10071010, 2009.

[18] J. Q. Mo and W. T. Lin, "Asymptotic solution of activator inhibitor systems for nonlinear reaction diffusion equations," Journal of Systems Science and Complexity, vol. 21, no. 1, pp. 119-128, 2008.

[19] J. Q. Mo and H. Wang, "Nonlinear singularly perturbed approximate solution for generalized Lotke-Volterra ecological model," Acta Ecologica Sinica, vol. 27, no. 10, pp. 4366-4370, 2007.

[20] J. Q. Mo, J. S. Yao, and H. Wang, "The nonlinear species group singularle perturbed Robin problems for reaction diffusion system," International Journal of Biomathematics, vol. 22, no. 2, pp. 193-199, 2007.

[21] J. Q. Mo and K. R. Zhou, "Singular perturbation for nonlinear species group reaction diffusion systems," International Journal of Biomathematics, vol. 21, no. 4, pp. 481-488, 2006.

[22] L. Hou, H. L. Li, J. J. Zhang, D. Z. Lin, and L. Qiu, "Boundarylayer eigen solutions for multi-field coupled equations in the contact interface," Applied Mathematics and Mechanics, vol. 31, no. 6, pp. 719-732, 2010.

[23] L. Hou and L. Qiu, "Computation and asymptotic analysis in the impact problem," Acta Mathematicae Applicatae Sinica, English Series, vol. 25, no. 1, pp. 117-126, 2009.

[24] Y. H. Feng and J. Q. Mo, "Asymptotic solution for singularly perturbed fractional order differential equation," International Journal of Mathematics, vol. 36, no. 2, pp. 239-245, 2016.

[25] Y. H. Feng, X. F. Chen, and J. Q. Mo, "The generalized interior shock layer solution of a class of nonlinear singularly perturbed reaction diffusion problem," Journal of Applied Mathematics, vol. 29, no. 1, pp. 161-165, 2016.

[26] Y. H. Feng and J. Q. Mo, "A class of soliton travelling wave solution for the nonlinear nonlocal disturbed LGH equation," Journal of Applied Mathematics and Mechanics, vol. 37, no. 4, pp. 426-433, 2016, (in Chinese).

[27] Y. H. Feng, L. F. Shi, Y. H. Xu, and J. Q. Mo, "The study for a class of atomy plasma diffusion model in atmosphere," Journal of Applied Mathematics and Mechanics, vol. 36, no. 6, pp. 639-650, 2015, (in Chinese).

[28] Y. H. Feng, X. F. Chen, and J. Q. Mo, "The shock wave solution of a class of singularly perturbed problem for generalized nonlinear reaction diffusion equation," Journal of Applied Mathematics and Mechanics, vol. 30, no. 1, pp. 1-7, 2017.

[29] J. H. He, "Variational approach for nonlinear oscillators," Chaos, Solitons \& Fractals, vol. 34, no. 5, pp. 1430-1439, 2007.

[30] Z. L. Tao and G. H. Chen, "An effective modification of JiHuan He's variational approach to nonlinear singular oscillator," Journal of Low Frequency Noise, Vibration and Active Control, vol. 38, no. 3-4, pp. 1023-1027, 2019.

[31] L. Barbu and G. Morosanu, Singularly Perturbed BoundaryValue Problems, Birkhauserm Verlag AG, Basel, Switzerland, 2007.
[32] E. M. de Jager and F. R. Jiang, The Theory of Singular Perturbation, North-Holland Publishing, Amsterdam, Netherlands, 1996. 